\title{
Time-dependent Behavior of Microvascular Blood Flow and Oxygenation: a Predictor of Functional Outcomes
}

\author{
Katarzyna Z. Kuliga, Rodney Gush, Geraldine F. Clough and Andrew J. Chipperfield*
}

\begin{abstract}
Objective: This study investigates the timedependent behaviour and algorithmic complexity of low frequency, periodic oscillations in blood flux and oxygenation signals from the microvasculature. Methods: Microvascular blood flux (BF) and oxygenation (OXY: oxyHb, deoxyHb, totalHb and $\mathrm{SO}_{2} \%$ ) was recorded from 15 healthy young adult males using combined laser Doppler fluximetry and white light spectroscopy with local skin temperature clamped to $33^{\circ} \mathrm{C}$ and during local thermal hyperaemia (LTH) at $43^{\circ} \mathrm{C}$. Power spectral density (PSD) of the BF and OXY signals was evaluated within the frequency range $(0.0095-1.6 \mathrm{~Hz})$. Signal complexity was determined using the Lempel-Ziv (LZ) algorithm. Results: Foldincrease in BF during LTH was $15.6(10.3,22.8)$ and in OxyHb 4.8 (3.5,5.9) (median, range). All BF and OXY signals exhibited multiple oscillatory components with clear differences in signal power distribution across frequency bands at $33^{\circ} \mathrm{C}$ and $43^{\circ} \mathrm{C}$. Significant reduction in the intrinsic variability and complexity of the microvascular signals during LTH was found, with mean LZ complexity of $B F$ and OxyHb falling by $25 \%$ and $49 \%$, respectively $(\mathbf{p}<0.001)$. Conclusion: These results provide corroboration that in human skin microvascular blood flow and oxygenation are influenced by multiple, time-varying oscillators that adapt to local influences and become more predictable during increased haemodynamic flow. Significance: Recent evidence strongly suggests that the inability of microvascular networks to adapt to an imposed stressor is symptomatic of disease risk which might be assessed via $B F$ and $O X Y$ via the combination signal analysis techniques described here.
\end{abstract}

Index Terms-Flow motion, power spectral density, Lempel Ziv complexity, microcirculation, skin, blood flow, tissue oxygenation, laser Doppler flowmetry, white light spectrometry.

\section{INTRODUCTION}

$\mathrm{T}^{\mathrm{H}}$ HE maintenance of an adequate blood flow through a vascular network sufficient to meet the metabolic

This work was supported in part by the NIHR/Wellcome Trust Southampton Clinical Research Facility. K. Z. Kuliga was supported by a UK EPSRC CASE PhD Studentship. The data for this work is accessible through the University of Southampton Institutional Research Repository at http//dx.doi.org/105258/SOTON/D0179.

K. Z. Kuliga and G. F. Clough are with Human Development and Health, Faculty of Medicine, University of Southampton, England. R. Gush is Moor Instruments Ltd., Axminster, England. *A. J. Chipperfield is with the Bioengineering Science Group, Faculty of Engineering and the Environment, University of Southampton, Highfield, Southampton, SO17 1BJ England (correspondence e-mail: a.j.chipperfield@soton.ac.uk).

Copyright (c) 2016 IEEE. Personal use of this material is permitted. However, permission to use this material for any other purposes must be obtained from the IEEE by sending an email to pubs-permissions@ieee.org. demands of the tissue is dependent on neural, humoral and local vaso-mechanisms that determine vascular tone and flow patterns within the microvasculature [1]. Recently, Frisbee and others [2] have shown that attenuation of these flow patterns may be a major contributor to disease risk. They argue that if we are to understand network functionality and flexibility in cardiovascular (CV) and metabolic (Met) disease it is important to obtain quantitative in vivo information on the temporal behaviour and spatial distribution of microvascular perfusion.

Time series analysis has been extensively used to describe the dynamic and non-linear characteristics of biological data. Such signals include electroencephalogical (EEG) and electrocardiographic (ECG) signals [3] as well as those from nocturnal oximetry [4]. Signals obtained from superficial vascular networks using Laser Doppler (LD) flowmetry have also been characterised using time series analysis. They have been shown to contain spontaneous, rhythmic oscillatory fluctuations in blood flux (BF), termed microvascular flowmotion [5]. The oscillations are of both local and systemic origin, with low frequency (LF) oscillations arising from the influence on vascular tone of local myogenic $(\sim 0.06-0.15$ $\mathrm{Hz})$, neurogenic $(\sim 0.02-0.06 \mathrm{~Hz})$ and endothelial $(\sim 0.0095-$ $0.02 \mathrm{~Hz}$ ) activity [6-8]. Additionally, the higher frequency (HF) oscillatory haemodynamic effects of the heart beat $(\sim 0.4-1.6 \mathrm{~Hz})$ and respiratory activity $(\sim 0.15-0.4 \mathrm{~Hz})$ may also be transmitted to the microcirculation.

Variations in the amplitude and relative contribution of these flowmotion oscillations have been associated with a decline in microvascular function in individuals at risk of $\mathrm{CV}$ and Met disease [9-13] (for review see [14]). Studies in a primate model of diabetes have shown a reduced complexity of the BF signal using a Lempel-Ziv (L-Z) complexity algorithm [15]. While in humans with a familial predisposition to hypertension, altered microvascular haemodynamics have been associated with diminished chaotic ischaemic flow [16]. More recently, chaotic network attractor analysis [17, 18] has revealed a declining adaptability of microvascular flow patterns in rodent models of $\mathrm{CV}$ and Met disease [2]. However, the relationship between the non-linear oscillatory dynamics of the BF signal, microvascular network perfusion heterogeneity and adaptability (and their impact on microvascular pathology) has yet to be fully elucidated.

An adequate and appropriate network perfusion is essential 
for tissue oxygenation and to meet tissue demand. Several theoretical models of microvascular network perfusion control have explored the importance of spatial and temporal variations in blood perfusion and haemoglobin concentration to oxygen delivery to the tissue [19]. Recent studies in vivo using time series analysis of simultaneously recorded skin blood flux (BF) and oxygenation (OXY) have shown that these signals oscillate over broad, generally similar frequency ranges, with varying total power and relative contribution from low and high frequency power spectral density (PSD) bands $[20,21]$. They have further shown that the oscillatory properties of blood flux and oxygenation may influence oxygen extraction [22, 23]. It remains unclear, however, whether alterations in perfusion complexity and flowmotion control may have functional significance for oxygen delivery and exchange within the microvasculature.

Our aim in the current study was to undertake time series analysis to determine the spectral power and complexity of oscillatory rhythms in continuously and simultaneously acquired microvascular blood flow and oxygenation signals in the skin. Data sets were obtained under two stable haemodynamic conditions: resting - where local skin temperature was clamped at $33^{\circ} \mathrm{C}$, and during local thermal hyperaemia (LTH) - vasodilatation to local warming of the skin to $43^{\circ} \mathrm{C}$. Under these controlled conditions we sought to identify the differential temporal and spectral behaviour of the oscillatory processes influencing microvascular blood flow and oxygenation and to determine their impact on network function and flexibility.

\section{MAterials AND MethodS}

\section{A. Study Design}

All participants were non-smokers and refrained from caffeine-containing drinks and food for at least 2 hours and strenuous exercise for 24 hours, before testing. None of the participants were taking any medications.

Studies were performed in a temperature-controlled room maintained at between $23 \cdot 0-23.5^{\circ} \mathrm{C}$. All participants were acclimatised for $30 \mathrm{~min}$ prior to testing. Measurements were made with the participant sitting comfortably in a reclining blood infusion chair with their arm supported at heart level.

The study was approved by Research Ethics Committee of University of Southampton and Southampton General Hospital (REC Number: SOMSEC091.10; RHMMED0992). The study was performed in accordance with standards set by the Declaration of Helsinki. All participants gave written informed consent. All data supporting this study are openly available from the University of Southampton repository at http://dx.doi.org/10.5258/SOTON/D0179.

\section{B. Capture of Laser Doppler and OXY Signals}

Skin microvascular blood flux (BF), oxygenation (OXY) and temperature were measured simultaneously using a combined laser Doppler (LD) and white light reflectance probe (Moor CP7-1000 blunt needle probe, Moor Instruments Ltd, Axminster, UK) with an LD fibre separation of $0.5 \mathrm{~mm}$ and $\mathrm{SO}_{2}$ separation of $1 \mathrm{~mm}$, using a single point $785 \mathrm{~nm}, 1$ $\mathrm{mW}$ low power red laser light source (moorVMS-LDF2, Moor Instruments Ltd, UK) and a 400-700 nm, $<6 \mathrm{~mW}$ white light source (moorVMS-OXY, Moor Instruments Ltd, UK). The probe was mounted in a MoorVHP1 skin heating block with an active heating diameter of $11 \mathrm{~mm}$ controlled by the MoorVMS-HEAT skin heater. Skin temperature is measured by a miniaturised negative temperature coefficient thermistor built into the device controlling skin warming with a precision of $\pm 0.1^{\circ} \mathrm{C}$ and resolution of $0.1^{\circ} \mathrm{C}$. The heating block and probe were placed on the ventral surface of the non-dominant forearm using a double-sided sticky O-ring, approximately 10 $\mathrm{cm}$ from the wrist and avoiding visible veins.

$\mathrm{BF}$ and OXY signals were recorded continuously from the skin at ambient temperature and then with the temperature of the heating disc set to $33^{\circ} \mathrm{C}$. After a period of $20 \mathrm{~min}$ baseline recording, the heating disc was raised to $43^{\circ} \mathrm{C}$ at a rate of $15^{\circ} \mathrm{C}$ per min (change from $33^{\circ} \mathrm{C}$ to $43^{\circ} \mathrm{C}$ in $40 \mathrm{~s}$ ) and maintained at this temperature for a further $25 \mathrm{~min}$ recording period (Fig. 1). Signals were recorded at a sampling rate of 40 $\mathrm{Hz}$.

We have previously shown that the inter-individual coefficients of variation (CV) for resting $\mathrm{BF}$ and $\mathrm{SO}_{2}$ measured using the combined probe in a cohort of 10 healthy individuals at ambient room temperature was 0.15 and 0.09 , respectively [20] (CV values of $<0.35$ deemed as acceptable [24]). The inter-individual CVs for oxyHb, deoxyHb, and totalHb were $0.19,0.09$ and 0.10 , respectively $(\mathrm{n}=10)$. The intra-class correlation coefficients (ICC) for resting $\mathrm{BF}$ and $\mathrm{SO}_{2}$ was 0.85 (almost perfect) and 0.68 (substantial [25]), respectively $(\mathrm{n}=10)$. Monte Carlo modelling and coherence analysis of the $\mathrm{LDF}$ and $\mathrm{SO}_{2}$ measurements suggests that they sample to a similar depth in the same vascular network although this is not yet proven [20].

\section{Signal Analysis}

Time-domain $\mathrm{BF}$ was recorded in arbitrary perfusion units $(\mathrm{PU})$ and measures of oxyHb, deoxyHb and total $\mathrm{Hb}$ (total $\mathrm{Hb}=$ oxyHb + deoxyHb) in arbitrary units $(\mathrm{AU})$. $\mathrm{SO}_{2}$ in per cent (\%) was derived from the relationship $\mathrm{SO}_{2}=($ oxyHb/total $\mathrm{Hb})$ x $100 \%$ [20]. Values for BF and OXY parameters were determined over the last $5 \mathrm{~min}$ of each haemodynamic state using moorVMS-PC software (Moor Instruments Ltd, UK).

Frequency-domain Prior to spectral analysis signals were filtered using a zero-phase low-pass filter with $2 \mathrm{~Hz}$ cut-off, resampled to $10 \mathrm{~Hz}$ and detrended using a moving-average with a 200 second window to attenuate frequency content below $0.005 \mathrm{~Hz}$. Spectral density was estimated by Welch's method of Fourier transform with a Hanning window size of $200 \mathrm{~s}$ and $50 \%$ overlap between windows over continuous 10 min recording periods using MATLAB (R2014a, MathWorks, UK). The power contribution was evaluated within the frequency range $(0.0095-1.6 \mathrm{~Hz})$, divided into frequency intervals corresponding to endothelial $(0.0095-0.02 \mathrm{~Hz})$, sympathetic $(0.02-0.06 \mathrm{~Hz})$, myogenic $(0.06-0.15 \mathrm{~Hz})$, respiratory $(0.15-0.4 \mathrm{~Hz})$ and cardiac $(0.4-1.6 \mathrm{~Hz})$ activity [26]. Total spectral power was estimated as the sum of ed. However, permission to use this material for any other Copyright (c) 2016 IEEE. Personal use of this material is permitted. However, permission to u
purposes must be obtained from the IEEE by sending an email to pubs-permissions@ieee.org. 


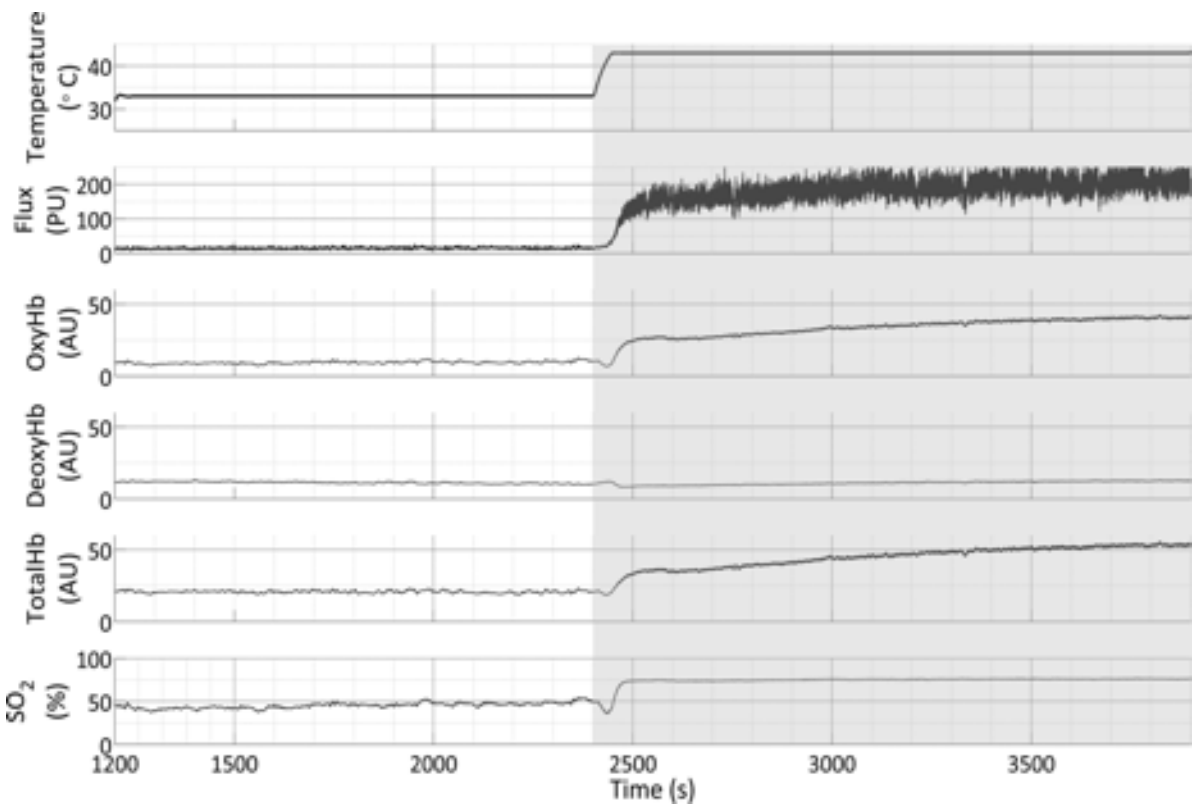

Fig. 1. Simultaneous outputs from the combined $\mathrm{LDF} / \mathrm{OXY}$ probe recorded in the skin at the ventral forearm in a healthy male at $33^{\circ} \mathrm{C}$ ambient skin temperature and during local warming to $43^{\circ} \mathrm{C}$ (grey area) showing: skin temperature; laser Doppler blood flux (BF) in arbitrary perfusion units (PU); oxygenated haemoglobin (oxyHb); deoxygenated haemoglobin (deoxyHb); total haemoglobin (totalHb = oxyHb + deoxyHb) (all in arbitrary units $\mathrm{AU}$ ); tissue oxygen saturation $\left(\mathrm{SO}_{2}=(\right.$ oxyHb/totalHb $\left.) \times 100 \%\right)$

absolute power across the five frequency intervals $(0.0095-1.6$ $\mathrm{Hz}$ ) and expressed in $\mathrm{U}^{2} / \mathrm{Hz}$. Power spectral density contribution (PSD contribution) was calculated relative to total spectral power and is expressed as a fraction between 0 and 1 .

Complexity In order to determine how much the BF and OXY signals differed from a random sequence [27] we calculated the Lempel-Ziv (LZ) complexity of the time series of $\mathrm{BF}$ and $\mathrm{OXY}$ signals over $10 \mathrm{~min}$ at $33^{\circ} \mathrm{C}$ and then $43^{\circ} \mathrm{C}$. The 10 min signal is divided into 15 epochs of length 40 seconds and converted from a time series to a sequence of zeros and ones. In Tigno et al. [27] this sequence is determined by comparing each element in the time series with the median value and replacing it with a zero if it is less than the median or a one otherwise. Here we have used a delta encoding method whereby a zero is recorded if a value is less than the previous value in the time series or a one otherwise as this captures more of the variability in the signals. Primitive LZ complexity is then calculated for each of these epochs to estimate the upper complexity limit. A LZ-complexity index was calculated as the mean of the $15 \times 40$ second epochs for each sampled signal.

We elected to focus on the OxyHb output as the prime OXY signal for complexity analysis. While $\mathrm{SO}_{2} \%$ tissue oxygenation (oxyHb/totalHb) x $100 \%)$ signal is indicative of the balance between $\mathrm{O}_{2}$ delivery and tissue $\mathrm{O}_{2}$ consumption in the tissue volume sampled and may thus be more 'physiologically informative', oxyHb as an underived output for signal processing. However, we saw similar trends in both oxyHb and $\mathrm{SO}_{2}$ signals.

\section{Statistical Analysis}

Statistical analysis was performed using GraphPad Prism (Prism 6, GraphPad Software, Inc., USA) and IBM SPSS Statistics 22 (IBM United Kingdom Limited, UK). Data were Copyright (c) 2016 IEEE. Personal use of this material is permitted. However, permission to use this material for any other purposes must be obtained from the IEEE by sending an email to pubs-permissions@ieee.org. tested for normal distribution using D'Agostino \& Pearson omnibus normality test and presented as either mean \pm standard deviation (SD) for normally distributed data or median with interquartile range (IQR) for non-normally distributed data. Normally distributed data were compared using a Students t-test and non-normally distributed data using a Mann-Whitney test. Pearson correlation coefficients are presented for univariate regression analysis of baseline data. In all cases a value of $\mathrm{p}<0.05$ was taken to indicate statistical significance.

\section{RESULTS}

Fifteen healthy males with a mean age $24.3 \pm 2.4 \mathrm{y}$ and BMI $23.7 \pm 2.8 \mathrm{~kg} \cdot \mathrm{m}^{-2}$ (mean \pm SD) participated in the study. The mean resting forearm skin temperature measured under ambient conditions was $31.2 \pm 1.0^{\circ} \mathrm{C}(\mathrm{n}=15)$.

Increased skin $B F$ and oxygenation in the time-domain during LTH An example of the raw $\mathrm{BF}$ and $\mathrm{OXY}$ signals obtained at $33^{\circ} \mathrm{C}$ and at $43^{\circ} \mathrm{C}$ is shown in Fig. 1. Mean values for $\mathrm{BF}$ and $\mathrm{OXY}$ parameters measured under the two haemodynamic conditions are summarized in Table I. Clamping the skin temperature at $33^{\circ} \mathrm{C}$ had no significant effect on any of the parameters compared with those measured at ambient temperature ( $\mathrm{p}$ all $>0.05$ ). Warming the skin to $43^{\circ} \mathrm{C}$ induced a sustained local thermal hyperaemia (LTH) with a fold increase in BF of $15.6(10.3,22.8)$ and oxyHb of $4.8(3.5,5.9)$ (median and IQR; $\mathrm{n}=15)$.

Total Hb (indicative of microvascular blood volume) more than doubled during LTH $(2.3(1.5,3.1)$ (median, IQR) $(n=15)$. There was a linear relationship between the reciprocal of $\mathrm{BF}$ (corrected for totalHb) and $\mathrm{SO}_{2}$ measured at $33^{\circ} \mathrm{C}$ and $43^{\circ} \mathrm{C}$ $\left(\mathrm{r}^{2}=0.69, \mathrm{p}<0.0001\right)[20]$. 

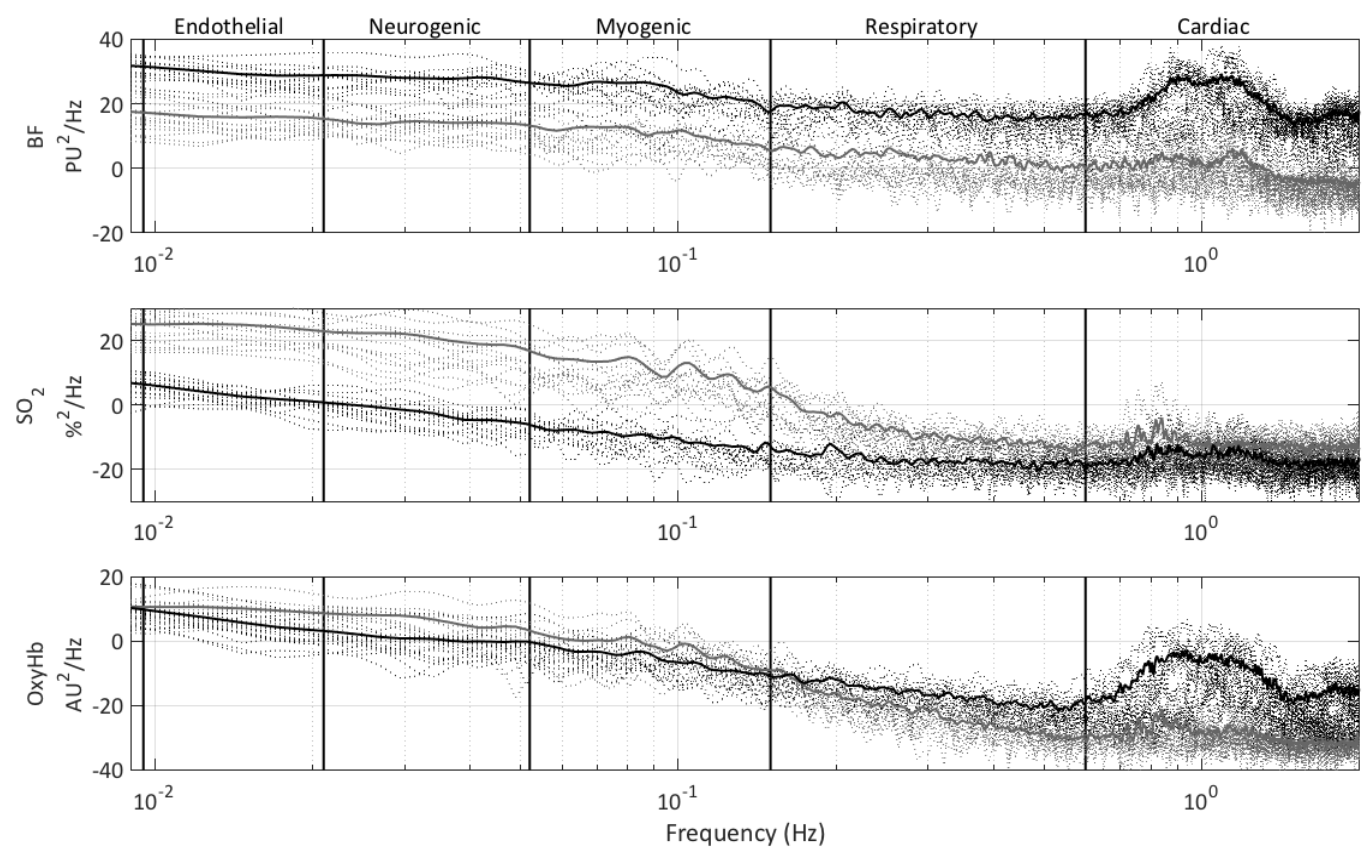

Fig. 2. Individual and mean PSD spectra of $\mathrm{BF}, \mathrm{SO}_{2}$ and oxyHb measured in the skin of 15 healthy participants at $33^{\circ} \mathrm{C}$ (grey) and $43^{\circ} \mathrm{C}$ (black). Individual spectra shown as dotted lines and mean as solid lines for $\mathrm{n}=15$ individuals.

Altered distribution of signal power in skin BF and oxygenation in the frequency-domain during LTH All signals recorded at $33^{\circ} \mathrm{C}$ and $43^{\circ} \mathrm{C}$ for all 15 healthy male participants exhibited multiple oscillatory components as shown in Fig. 2. The vasodilatation to local warming of the skin to $43^{\circ} \mathrm{C}$ resulted in a significant increase in absolute total power of the oscillations in the BF signal $(\mathrm{p}<0.0001)$ which was strongly correlated with $\mathrm{BF}\left(\mathrm{r}^{2}=0.78, \mathrm{p}<0.0001\right)$. There was no significant change in absolute total power of the oscillations in the oxyHb signal during LTH $(\mathrm{p}=0.34)$. Absolute oscillatory power in the calculated $\mathrm{SO}_{2}$ signal decreased during LTH $(\mathrm{p}<0.0001)$. This was largely as a result of the fall in deoxyHb signal power $(\mathrm{p}=0.025)$ (Fig. 3) occurring in the LF bands as described below.

While the low frequency power/frequency spectra appear to lack discernible peaks within each frequency band there are clear differences in the distribution of signal power across the frequency bands in the BF and OXY signals measured at $33^{\circ} \mathrm{C}$ and $43^{\circ} \mathrm{C}$. The relative PSD contributions $(0-1)$ of the five frequency bands to all signals are shown in Table II. At $33^{\circ} \mathrm{C}$ the majority of the signal power resided within the LF endothelial, neurogenic and myogenic bands. During temperature-induced vasodilatation the relative PSD contribution of the high frequency (HF) cardiac band increased in all signals (Table II). The increase was most marked in the BF signal, indicative of greater modulation of this signal by heartbeat. The PSD contribution of all three LF energy bands in both BF and OXY signals fell during LTH as the contribution of the cardiac $(\sim 1 \mathrm{~Hz})$ band increased (Table III).

Decreased complexity of skin BF and oxygenation signals during $\boldsymbol{L T H}$ We were able to estimate LZ-complexity of BF and OXY signals in both haemodynamic states in 14 of the 15 participants. LZ-complexity of BF and OXY signals showed

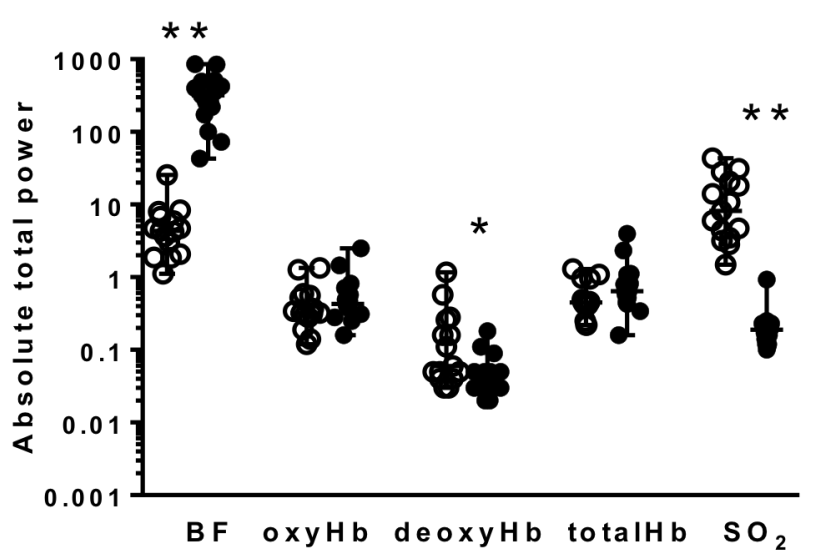

Fig. 3. Absolute total power in BF and OXY signals (oxyHb, deoxyHb, total $\mathrm{Hb}$ and $\mathrm{SO}_{2}$ ) measured in the skin of healthy male participants during local thermal warming from $33^{\circ} \mathrm{C}$ (open circles) and $43^{\circ} \mathrm{C}$ (closed circles). Data are median and range, $\mathrm{n}=15 .{ }^{*} \mathrm{p}<0.05$ and $* * \mathrm{p}<0.000133^{\circ} \mathrm{C}$ vs $43^{\circ} \mathrm{C}$.

TABLE I

MEASURED BF AND OXY PARAMETERS

\begin{tabular}{llllll}
\hline \hline & \multicolumn{1}{c}{$\begin{array}{c}\mathrm{BF} \\
(\mathrm{PU})\end{array}$} & $\begin{array}{c}\text { OxyHb } \\
(\mathrm{AU})\end{array}$ & $\begin{array}{l}\text { deOxyHb } \\
(\mathrm{AU})\end{array}$ & $\begin{array}{l}\text { totalHb } \\
(\mathrm{AU})\end{array}$ & $\begin{array}{l}\mathrm{SO}_{2} \\
(\%)\end{array}$ \\
\hline $33^{\circ} \mathrm{C}$ mean & 13.2 & 6.2 & 8.9 & 14.1 & 41.9 \\
$(\mathrm{SD})$ & $(4.9)$ & $(2.9)$ & $(3.7)$ & $(4.9)$ & $(11.5)$ \\
& & & & & \\
$43^{\circ} \mathrm{C}$ mean & 215.3 & 33.3 & 11.8 & 45.1 & 73.1 \\
$(\mathrm{SD})$ & $(65.0)^{* *}$ & $(10.9)^{* *}$ & $(2.7)^{* *}$ & $(13.5)^{*}$ & $(3.1)^{* *}$ \\
$\begin{array}{l}\text { Fold Change } \\
\text { Median }\end{array}$ & 15.6 & 4.8 & 0.6 & 2.3 & 0.6 \\
$(\mathrm{IQR})$ & $(10.3-22.8)$ & $(3.5-5.9)$ & $(0.1-1.1)$ & $(1.5-3.1)$ & $(0.5-1.3)$ \\
\hline \hline
\end{tabular}

Microvascular blood flux (BF in arbitrary perfusion units PU) and oxygenation parameters [oxygenated haemoglobin (oxyHb); deoxygenated haemoglobin (deoxyHb); total haemoglobin (totalHb $=$ oxyHb + deoxyHb) (all in arbitrary units $\mathrm{AU})$ and tissue oxygen saturation $\left(\mathrm{SO}_{2}=\right.$ $(\mathrm{oxyHb} /$ totalHb) $\times 100 \%)$ ] measured in the skin at the forearm of 15 healthy male participants at $33^{\circ} \mathrm{C}$ and then $43^{\circ} \mathrm{C}$. ${ }^{*} \mathrm{p}<0.05 * * \mathrm{p}<0.0001$.

Copyright (c) 2016 IEEE. Personal use of this material is permitted. However, permission to use this material for any other purposes must be obtained from the IEEE by sending an email to pubs-permissions@,iee.org. 
TABLE II

PSD CONTRIBUTIONS IN BF AND OXY SIGNAL ACROSS FIVE FREQUENCY BANDS RECORDED AT THE FOREARM AT $33^{\circ} \mathrm{C}$ AND $43^{\circ} \mathrm{C}$.

\begin{tabular}{|c|c|c|c|c|c|c|}
\hline Signal & & Endothelial & Neurogenic & Myogenic & Respiratory & Cardiac \\
\hline$\overline{B F}$ & $\begin{array}{l}33^{\circ} \mathrm{C} \\
43^{\circ} \mathrm{C}\end{array}$ & $\begin{array}{l}0.10(0.05) \\
0.04(0.02)^{*}\end{array}$ & $\begin{array}{l}0.22(0.8) \\
0.08(0.04)^{*}\end{array}$ & $\begin{array}{l}0.20(0.06) \\
0.08(0.03)^{*}\end{array}$ & $\begin{array}{l}0.12(0.08) \\
0.05(0.03)^{*}\end{array}$ & $\begin{array}{l}0.37(0.09) \\
0.76(0.07)^{*}\end{array}$ \\
\hline oxyHb & $\begin{array}{l}33^{\circ} \mathrm{C} \\
43^{\circ} \mathrm{C}\end{array}$ & $\begin{array}{l}0.37(0.13) \\
0.20(0.13)^{*}\end{array}$ & $\begin{array}{l}0.43(0.11) \\
0.17(0.21)^{*}\end{array}$ & $\begin{array}{l}0.17(0.11) \\
0.10(0.06)^{*}\end{array}$ & $\begin{array}{l}0.02(0.02) \\
0.04(0.03)^{*}\end{array}$ & $\begin{array}{l}0.01(0.01) \\
0.49(0.18)^{*}\end{array}$ \\
\hline deoxyHb & $\begin{array}{l}33^{\circ} \mathrm{C} \\
43^{\circ} \mathrm{C}\end{array}$ & $\begin{array}{l}0.44(0.17) \\
0.26(0.16)^{*}\end{array}$ & $\begin{array}{l}0.37(0.11) \\
0.18(0.08)^{*}\end{array}$ & $\begin{array}{l}0.12(0.08) \\
0.07(0.03)^{*}\end{array}$ & $\begin{array}{l}0.03(0.03) \\
0.05(0.03)\end{array}$ & $\begin{array}{l}0.04(0.02) \\
0.44(0.17)^{*}\end{array}$ \\
\hline totalHb & $\begin{array}{l}33^{\circ} \mathrm{C} \\
43^{\circ} \mathrm{C}\end{array}$ & $\begin{array}{l}0.35(0.14) \\
0.20(0.15)^{*}\end{array}$ & $\begin{array}{l}0.43(0.10) \\
0.16(0.07)^{*}\end{array}$ & $\begin{array}{l}0.19(0.10) \\
0.10(0.06)^{*}\end{array}$ & $\begin{array}{l}0.03(0.02) \\
0.04(0.03)\end{array}$ & $\begin{array}{l}0.01(0.01) \\
0.50(0.19)^{*}\end{array}$ \\
\hline $\mathrm{SO}_{2}$ & $\begin{array}{l}33^{\circ} \mathrm{C} \\
43^{\circ} \mathrm{C}\end{array}$ & $\begin{array}{l}0.32(0.14) \\
0.30(0.13)^{*}\end{array}$ & $\begin{array}{l}0.40(0.11) \\
0.23(0.09)^{*}\end{array}$ & $\begin{array}{l}0.14(0.09) \\
0.08(0.04)^{*}\end{array}$ & $\begin{array}{l}0.02(0.02) \\
0.06(0.03)^{*}\end{array}$ & $\begin{array}{l}0.02(0.01) \\
0.33(0.14)^{*}\end{array}$ \\
\hline
\end{tabular}

Data are mean and SD for $\mathrm{n}=15$ healthy male participants. ${ }^{*} \mathrm{p}<0.0543^{\circ} \mathrm{C}$ vs $33^{\circ} \mathrm{C}$

\section{A. Blood Flux}

B. $\mathrm{OxyHb}$
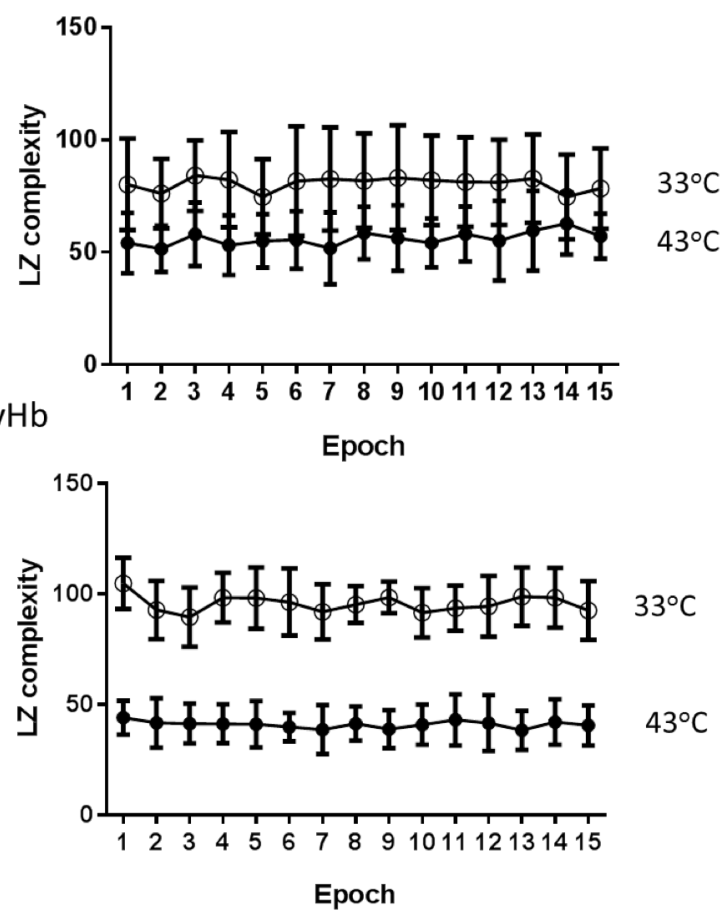

C.

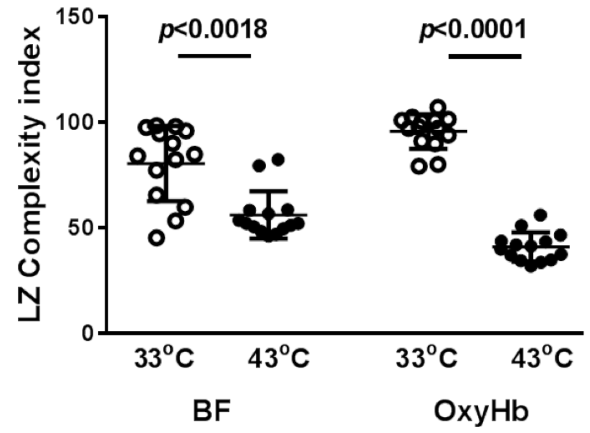

Fig. 4. Changes in Lempel-Ziv (LZ) complexity with local thermal warming of the skin. Epoch length $40 \mathrm{sec}$. A. Microvascular blood flux (BF), B. Microvascular oxygenated haemoglobin $(\mathrm{OxyHb})$. C. BF and OxyHb LZ complexity index expressed as mean value across the 15 epochs Open circles $33^{\circ} \mathrm{C}$, filled circles $43^{\circ} \mathrm{C}$. Data are mean $\pm \mathrm{SD}$ for $\mathrm{n}=14$ healthy participants.

relatively consistent variability over the 15 epochs in both haemodynamic states (Fig. 4A and B). However, signals appeared less variable and had fewer unique local states (a lower LZ-complexity) during LTH compared with the signals Copyright (c) 2016 IEEE. Personal use of this material is permitted. However, permission to use this material for any other purposes must be obtained from the IEEE by sending an email to pubs-permissions@ieee.org.
TABLE III

PSD CONTRIBUTION TO BF AND OXY CALCULATED ACROSS LF BANDS (ENDOTHELIAL, NEUROGENIC AND MYOGENIC).

\begin{tabular}{llll}
\hline \hline & \multicolumn{1}{c}{ Endothelial } & Neurogenic & Myogenic \\
& $0.0095-0.02 \mathrm{~Hz}$ & $0.02-0.06 \mathrm{~Hz}$ & $0.06-0.15 \mathrm{~Hz}$ \\
\hline $33^{\circ} \mathrm{C}$ & & & \\
$\mathrm{BF}$ & $0.19(0.09)$ & $0.42(0.10)$ & $0.39(0.13)$ \\
oxyHb & $0.38(0.13)$ & $0.44(0.11)$ & $0.18(0.11)$ \\
deoxyHb & $0.47(0.17)$ & $0.40(0.11)$ & $0.13(0.08)$ \\
totalHb & $0.36(0.14)$ & $0.44(0.10)$ & $0.19(0.11)$ \\
$\mathrm{SO}_{2}$ & $0.44(0.14)$ & $0.41(0.11)$ & $0.15(0.10)$ \\
$43^{\circ} \mathrm{C}$ & & & \\
$\mathrm{BF}$ & $0.20(0.09)$ & $0.40(0.13)$ & $0.40(0.14)$ \\
oxyHb & $0.41(0.17)$ & $0.37(0.13)$ & $0.22(0.09)$ \\
deoxyHb & $0.49(0.17)$ & $0.37(0.11)$ & $0.15(0.09)$ \\
totalHb & $0.40(0.19)$ & $0.37(0.13)$ & $0.22(0.11)$ \\
$\mathrm{SO}_{2}$ & $0.48(0.14)$ & $0.37(0.12)$ & $0.15(0.08)$ \\
\hline \hline
\end{tabular}

Data are mean and $\mathrm{SD}$ for $\mathrm{n}=15$ healthy male participants.

obtained at $33^{\circ} \mathrm{C}$. The $\mathrm{BF}$ LZ-complexity index (mean value across the 15 epochs for each individual) fell from $80.5 \pm 17.8$ to $56.1 \pm 11.2(\mathrm{p}=0.0018, \mathrm{n}=14)$ during warming (Fig. $4 \mathrm{C})$. A similar decline in LZ-complexity index was seen in the oxyHb signal $\left(33^{\circ} \mathrm{C}, 95.7 \pm 8.2 ; 43^{\circ} \mathrm{C} 40.9 \pm 6.9, \mathrm{p}<0.0001\right)$ (Fig. $4 \mathrm{C}$ ). LZ-complexity index of the $\mathrm{SO}_{2}$ signal at $33^{\circ} \mathrm{C}$ and $43^{\circ} \mathrm{C}$ was 103.0 \pm 4.8 and $95.4 \pm 9.1$, respectively $(\mathrm{p}=0.0004)$.

\section{DISCUSSION}

We set out to define the time-dependent behaviour of low frequency, periodic oscillations in terms of the spectral power and complexity of the oscillatory rhythms within simultaneously recorded microvascular blood perfusion and oxygenation signals. These low frequency oscillatory rhythms have been shown to be associated with local flowmotion control of network perfusion and to vary under physiological and pathophysiological conditions. Our findings provide strong evidence that in human skin, microvascular blood flux and oxygenation are similarly entrained by local influences, and that up to three quarters of the variation in the low frequency flow motion activity in the BF signal is linearly related to oxygenation. They further show that in healthy individuals there is the potential for adaptability of flowmotion and that flowmotion becomes more 
predictable/less variable in a high flow state; in this case a hyperaemia induced by local thermal warming.

\section{A. Network perfusion and oxygenation in low vs high flow} states in the time domain

The stable haemodynamic conditions under which time series analysis data were collected were initially when the skin was clamped at $33^{\circ} \mathrm{C}$ and network perfusion held at a basal level (i.e. under mild constrictor tone [28]), and subsequently during a sustained local hyperaemia induced by locally warming of the skin to $43^{\circ} \mathrm{C}$. Local warming evokes a biphasic response, with an initial transient (2-3 min) peak which has been shown to be largely mediated by a sensory nerve axon reflex [29]. This is followed by a prolonged, predominantly endothelial NO-mediated plateau $[30,31]$ that provides the more stable and sustained differential output for time-frequency analysis. The plateau is of sufficient duration to allow capture of more than the $2-5$ cycles $(3.5-8.75 \mathrm{~min})$ required for evaluation of the $0.01 \mathrm{~Hz}$ frequency band and the 10 min sampling period consistent with that used by other researchers (e.g. $[11,13,25])$.

In the current study, the warming-induced increase in BF ( $\sim 15$-fold) was similar to that we have reported previously in healthy forearm skin [32]. The LD blood flux signal is derived from both the number and velocity of red blood cells beneath the probe. Thus the increase in LD BF may be attributed to an increased total blood flow and/or greater blood volume through capillary recruitment. The increase in BF in the time domain was associated with an increase in total spectral energy over the range $0.0095-1.6 \mathrm{~Hz}$ of more than 33 -fold. The increase in tissue oxygenation and total spectral energy in the OXY signals during enhanced microvascular perfusion were less marked. This is perhaps unsurprising as healthy skin has a relatively low metabolic requirement and during LTH the increase in $\mathrm{BF}$ and thence $\mathrm{O}_{2}$ delivery far exceeds $\mathrm{O}_{2}$ demand by the tissue in a heightened metabolic state [20].

\section{B. Power-frequency profiles in low vs high flow states}

For a given individual, the power-frequency profiles of the simultaneously recorded $\mathrm{BF}$ and $\mathrm{OXY}$ signals at $33^{\circ} \mathrm{C}$ and $43^{\circ} \mathrm{C}$ shown in Fig. 2 did not overlap and were located at different power levels in the frequency intervals. This we take as indicative of an altered flowmotion activity between the two haemodynamic states as one power level cannot be obtained from a simple scaling of the other. It requires a more complex transformation of the oscillatory components. PSD analysis revealed differences in the relative contribution of the LF (endothelial, neurogenic and myogenic) and $\mathrm{HF}$ (respiratory and cardiac) components to the BF and oxygenation signals at the two haemodynamic states. When normalised to baseline $\left(33^{\circ} \mathrm{C}\right)$, the most marked increase during LTH in all signals was in the heart beat activity. The relative PSD contribution of the $\sim 1 \mathrm{~Hz}$ band in the BF signal increased from $37 \%$ at $33^{\circ} \mathrm{C}$ to $76 \%$ during warming-induced vasodilatation (Table II). It was similarly increased in all of the OXY signals. The increase in PSD in the $\sim 1 \mathrm{~Hz}$ band is consistent with a strong modulation of the signals as a result of the conduction of the cardiac-generated rhythm further through the vasodilated microvascular network. It may also be due to arteriovenous shunting of blood within the superficial dermal layers [33]. The contrary is observed during coolinginduced vasoconstriction in the skin, where normalised spectral energy in the cardiac band decreases [34].

We saw a reduction in the PSD contribution in all three LF energy bands during LTH in BF and OXY signals. The reduction in the normalised spectral energy in the neurogenic and myogenic bands during warming is consistent with withdrawal of myogenic and sympathetic activity to reduce vascular tone. Sheppard et al [34] using wavelet based analysis similarly reported a decrease in the myogenic band in the hand during warming, as did Gryglewska et al [35] using a Fast Fourier Transform (FFT). However, Hodges et al [36] also using FFT reported warming-induced increases in the three LF bands at both the forearm and the leg in young healthy individuals. The reasons for this discrepancy are unknown but may be site and/or heating-rate dependent [37]. The reduction in $\mathrm{PSD}$ contribution of the $\sim 0.01 \mathrm{~Hz}$ (endothelial) band of the BF signal that we report in the current study is contrary to that reported previously by us [9, 20] and others [36, 38]. Endothelium-derived NO has been shown to play an important role in the sustained LTH [39] and thus it might be expected that activity within the endotheliumrelated spectral band would increase. However, it is of interest to note that in the current study we analysed PSD across the five spectral frequency bands rather than the three LF bands used in the studies cited above. An increase in PSD contribution of the cardiac band during LTH will give rise to an apparent reduction in the relative PSD contribution of the LF bands. The HF bands were therefore excluded and the relative PSD contribution of the remaining LF bands to the BF and oxyHb signals calculated as shown in Table III. The LF distribution across the three LF bands was not significantly changed during LTH in healthy forearm skin. Thus to what extent the direction of change in LTH-induced LF flowmotion activity is process driven has yet to be fully elucidated.

\section{Complexity of network perfusion and oxygenation in low vs high flow states}

Algorithmic complexity of time series data provides a measure of the non-random structure or predictability of the time series. Generally, the LZ-complexity is derived from a transformation of the original signal into a binary sequence by comparing each value with a pre-set threshold such as the mean or median value as in [40]. However, this can be associated with a loss of essential characteristics from the signal dynamics [41]. Hence we have used delta encoding to track the signal trajectory rather than value relative to a fixed reference at each time point. As Lempel and Ziv [42] describe, the complexity of the signal can more realistically be measured by the primitive method. This gives an upper bound on complexity and accounts for all the steps in the exhaustive method (lower bound). Using this approach we have shown that the variability of the BF and OXY signals decreases in a state where the skin vasculature approaches maximal 
dilatation and network perfusion. It is further possible that the reduction in complexity of the BF and OXY signals during increased haemodynamic flow may be attributable to the increase in the $\sim 1 \mathrm{~Hz}$ periodic component or its synchronisation across the microvascular network.

The decline in randomness during local warming that we see in healthy human forearm skin is consistent with that reported by Tigno et al. [15] in the skin of primates. These authors have also reported that the LZ-complexity of the BF signal reduces with disease severity and is more than $30 \%$ lower in primates with type 2 diabetes than their normoglycaemic controls [15].

Greater variability in patterns in blood flowmotion is thought to give rise to more effective microvascular network perfusion [43]. Greater variability may also indicate an ability to influence flow independently of the spectral amplitude of flowmotion [16]. Thus a declining spontaneous variation in flowmotion activity as seen in vascular beds at risk of $\mathrm{CV}$ and Met disease [14] may contribute to a loss of system flexibility and adaptability in the face of physiological or pathological challenge.

While the metabolism in skin is low and oxygen delivery far exceeds demand under resting conditions, we did see a strong linear correlation between $\mathrm{BF}$ and oxyHb LZcomplexity indices measured across the two haemodynamic states $(r=0.68, p<0.0001)$. Computational models of oxygen transport in the capillary network of skeletal muscle have shown that chaotic activity promotes more efficient oxygenation than simple periodic patterns of vasomotion [44]. This suggests that a declining variability in flowmotion signals may be disadvantageous to patient health. Further, the large difference in $\mathrm{OxyHb}$ complexity between $33^{\circ} \mathrm{C}$ and $43^{\circ} \mathrm{C}$ suggests that this measure may be valuable in a clinical setting; for example in detecting early signs of sepsis or response to treatment in patients on intensive care.

\section{LIMITATIONS}

While this study reveals novel understanding of the potential for time series analysis of simultaneously recorded blood flux and oxygenation, it may also be subject to some limitations. A major strength of the study is that LTH has been widely used as a test to assess microvascular reactivity $[29,36,45]$ and a reduced cutaneous endothelium-mediated vasodilatation during LTH has been reported in CV and Met disease $[9,46,47]$. We have thus characterised the timedependent behaviour of microvascular blood flow and oxygenation under dynamic testing conditions that may be relevant to future studies in patient cohorts.

The power-frequency profiles that we report lacked visible peaks in the LF bands, i.e. the oscillations within a defined band lacked a dominant frequency. In contrast, the higher frequency cardiac band exhibited a clear peak around $1 \mathrm{~Hz}$, consistent with a resting heart rate of $\sim 60$ beats per minute. The borders of the frequency bands that we used were those defined by others and associated with local and systemic physiological activity [5]. It is possible that the LF profiles obtained in our healthy male cohort comprised multiple frequency components and that particularly in an enhanced haemodynamic state, the principal frequency component in a given band varied in time. We were unable to explore this using the FFT because it cannot be localised in time unlike the wavelet transform. It is also probable that the limits of the frequency bands will differ between individuals and under pathophysiological conditions.

\section{CONCLUSIONS}

Time and frequency domain methods alone have so far been unable to produce a robust and consistent description of the dynamics within the microcirculation in either healthy individuals during application of a standard stressor or in patient cohorts. The combination of time, frequency and complexity analysis offers an extended assessment of microvascular network behavior to inform how a loss of system flexibility may prevent microvascular networks from effectively adapting to an imposed stressor. As attenuation of flowmotion patterns may be a major contributor to disease risk, quantitative methods of analyzing temporal behavior are important if we are to understand network functionality and flexibility in cardiovascular and metabolic disease. This proof of concept study using LZ complexity analysis in healthy individuals under two imposed haemodynamic states needs now to be extended to patient cohorts.

\section{REFERENCES}

[1] M. Intaglietta, "Vasomotion and flowmotion: physiological and mechanisms and clinical evidence," Vascular Medicine Review, vol. 1, pp. 101-112, 1990.

[2] J. C. Frisbee, J. T. Butcher, S. J. Frisbee, I. M. Olfert, P. D. Chantler, L. E. Tabone, A. C. d'Audiffret, C. D. Shrader, A. G. Goodwill, P. A. Stapleton, S. D. Brooks, R. W. Brock, and J. H. Lombard, "Increased peripheral vascular disease risk progressively constrains perfusion adaptability in the skeletal muscle microcirculation," Am J Physiol Heart Circ Physiol, vol. 310, no. 4, pp. H488-504, Feb 15, 2016.

[3] A. M. Albano, B. P.D., C. C.J., X. T. Tigno, and P. E. Rapp, "Time series analysis, or the quest for qualitative measures of time dependent behavior," Phillipine Sciene Letters, vol. 1, no. 1, pp. 18-31, 2008.

[4] J. V. Marcos, R. Hornero, D. Alvarez, F. Del Campo, C. Zamarron, and M. Lopez, "Utility of multilayer perceptron neural network classifiers in the diagnosis of the obstructive sleep apnoea syndrome from nocturnal oximetry," Comput Methods Programs Biomed, vol. 92, no. 1, pp. 7989, Oct, 2008

[5] A. Stefanovska, M. Bracic, and H. D. Kvernmo, "Wavelet analysis of oscillations in the peripheral blood circulation measured by laser Doppler technique," IEEE Trans. Biomed. Eng, vol. 46, no. 10, pp. 1230-1239, 10/1999, 1999.

[6] P. Kvandal, S. A. Landsverk, A. Bernjak, A. Stefanovska, H. D. Kvernmo, and K. A. Kirkeboen, "Low-frequency oscillations of the laser Doppler perfusion signal in human skin," Microvasc Res, vol. 72, no. 3, pp. 120-7, Nov, 2006

[7] A. Colantuoni, S. Bertuglia, and M. Intaglietta, "Microvascular vasomotion: origin of laser Doppler flux motion," Int. J Microcirc. Clin. Exp, vol. 14, no. 3, pp. 151-158, 5/1994, 1994.

[8] T. Soderstrom, A. Stefanovska, M. Veber, and H. Svensson, "Involvement of sympathetic nerve activity in skin blood flow oscillations in humans," Am. J. Physiol Heart Circ. Physiol, vol. 284, no. 5, pp. H1638-H1646, 5/2003, 2003.

[9] M. R. Avery, D. Voegeli, C. D. Byrne, D. M. Simpson, and G. F. Clough, "Age and cigarette smoking are independently associated with the cutaneous vascular response to local warming," Microcirculation, vol. 16 , no. 8 , pp. 725-34, Nov, 2009.

Copyright (c) 2016 IEEE. Personal use of this material is permitted. However, permission to use this material for any other purposes must be obtained from the IEEE by sending an email to pubs-permissions@ieee.org. 
[10] G.F. Clough, V L'Esperance, M. Turzyniecka, L. Walter, A.J. Chipperfield, J. Gamble, A.J. Krentz, and C.D. Byrne. Functional dilator capacity is independently associated with insulin sensitivity and age in central obesity and is not improved by high dose statin treatment. Microcirculation, vol. 18(i), pp. 74-84, Jan 2011.

[11] M. Rossi, A. Carpi, M. C. Di, F. Franzoni, F. Galetta, and G. Santoro, "Skin blood flowmotion and microvascular reactivity investigation in hypercholesterolemic patients without clinically manifest arterial diseases," Physiol Res, vol. 58, no. 1, pp. 39-47, 2009, 2009.

[12] R. T. De Jongh, A. D. Clark, R. G. IJzerman, E. H. Serne, V. G. de, and C. D. Stehouwer, "Physiological hyperinsulinaemia increases intramuscular microvascular reactive hyperaemia and vasomotion in healthy volunteers," Diabetologia, vol. 47, no. 6, pp. 978-986, 6/2004, 2004.

[13] R. S. Bruning, W. L. Kenney, and L. M. Alexander, "Altered skin flowmotion in hypertensive humans," Microvasc Res, vol. 97, pp. 81-7, Jan, 2015.

[14] G. F. Clough, K. Z. Kuliga, and A. J. Chipperfield, "Flow motion dynamics of microvascular blood flow and oxygenation - evidence of adaptive changes in obesity and type 2 diabetes mellitus/insulin resistance," Microcirculation, Nov 3, 2016.

[15] X. T. Tigno, B. C. Hansen, S. Nawang, R. Shamekh, and A. M. Albano, "Vasomotion Becomes Less Random as Diabetes Progresses in Monkeys," Microcirculation, vol. 18, no. 6, pp. 429-439, Aug, 2011.

[16] B. Gryglewska, M. Necki, M. Zelawski, M. Cwynar, T. Baron, M. Mrozek, and T. Grodzicki, "Fractal dimensions of skin microcirculation flow in subjects with familial predisposition or newly diagnosed hypertension," Cardiol J, vol. 18, no. 1, pp. 26-32, 2011.

[17] D. Parthimos, D. H. Edwards, and T. M. Griffith, "Comparison of chaotic and sinusoidal vasomotion in the regulation of microvascular flow," Cardiovasc Res, vol. 31, no. 3, pp. 388-99, Mar, 1996.

[18] R. K. Pradhan, V. S. Chakravarthy, and A. Prabhakar, "Effect of chaotic vasomotion in skeletal muscle on tissue oxygenation," Microvasc Res, vol. 74, no. 1, pp. 51-64, Jul, 2007.

[19] T. W. Secomb, "Theoretical models for regulation of blood flow," Microcirculation, vol. 15, no. 8, pp. 765-75, Nov, 2008.

[20] K. Z. Kuliga, E. F. McDonald, R. Gush, C. Michel, A. J. Chipperfield, and G. F. Clough, "Dynamics of microvascular blood flow and oxygenation measured simultaneously in human skin," Microcirculation, vol. 21, no. 6, pp. 562-73, Aug, 2014.

[21] A. S. A. M. P. Bernjak, "Coherence between fluctuations in blood flow and oxygen saturation," Fluctuation and Noise Letters, vol. 11, no. 1, pp. 12, 2012.

[22] C. E. Thorn, H. Kyte, D. W. Slaff, and A. C. Shore, "An association between vasomotion and oxygen extraction," Am. J. Physiol Heart Circ. Physiol, vol. 301, no. 2, pp. H442-H449, 8/2011, 2011.

[23] C. E. Thorn, and A. C. Shore, "The role of perfusion in the oxygen extraction capability of skin and skeletal muscle," Am J Physiol Heart Circ Physiol, vol. 310, no. 10, pp. H1277-84, May 15, 2016.

[24] M. Roustit, S. Blaise, C. Millet, and J. L. Cracowski, "Reproducibility and methodological issues of skin post-occlusive and thermal hyperemia assessed by single-point laser Doppler flowmetry," Microvasc Res, vol. 79, no. 2, pp. 102-8, Mar, 2010.

[25] J. Svalestad, S. Hellem, G. Vaagbo, A. Irgens, and E. Thorsen, "Reproducibility of transcutaneous oximetry and laser Doppler flowmetry in facial skin and gingival tissue," Microvasc Res, vol. 79, no. 1, pp. 29-33, Jan, 2010.

[26] H. D. Kvernmo, A. Stefanovska, K. A. Kirkeboen, and K. Kvernebo, "Oscillations in the human cutaneous blood perfusion signal modified by endothelium-dependent and endothelium-independent vasodilators," Microvasc. Res, vol. 57, no. 3, pp. 298-309, 5/1999, 1999.

[27] X. T. Tigno, B. C. Hansen, and A. M. Albano, "Spectral Properties of Basal Vasomotion Predict Metabolic Risk Groups in Nonhuman Primates," Circulation, vol. 119, no. 10, pp. E291-E291, Mar 17, 2009.

[28] J. M. Johnson, C. T. Minson, and D. L. Kellogg, Jr., "Cutaneous vasodilator and vasoconstrictor mechanisms in temperature regulation," Compr Physiol, vol. 4, no. 1, pp. 33-89, Jan, 2014.

[29] C. T. Minson, "Thermal provocation to evaluate microvascular reactivity in human skin," J Appl Physiol (1985), vol. 109, no. 4, pp. 1239-46, Oct, 2010.
[30] V. E. Brunt, and C. T. Minson, "KCa channels and epoxyeicosatrienoic acids: major contributors to thermal hyperaemia in human skin," $J$ Physiol, vol. 590, no. 15, pp. 3523-34, Aug 1, 2012.

[31] V. E. Brunt, and C. T. Minson, "Cutaneous thermal hyperemia: more than skin deep," J Appl Physiol (1985), vol. 111, no. 1, pp. 5-7, Jul, 2011.

[32] G. Clough, A. Chipperfield, C. Byrne, M. F. de, and R. Gush, "Evaluation of a new high power, wide separation laser Doppler probe: potential measurement of deeper tissue blood flow," Microvasc. Res, vol. 78, no. 2, pp. 155-161, 9/2009, 2009.

[33] I. M. Braverman, "The cutaneous microcirculation," J Investig. Dermatol Symp. Proc, vol. 5, no. 1, pp. 3-9, 12/2000, 2000.

[34] L. W. Sheppard, V. Vuksanovic, P. V. McClintock, and A. Stefanovska, "Oscillatory dynamics of vasoconstriction and vasodilation identified by time-localized phase coherence," Phys Med Biol, vol. 56, no. 12, pp. 3583-601, Jun 21, 2011.

[35] B. Gryglewska, M. Necki, M. Cwynar, T. Baron, and T. Grodzicki, "Local heat stress and skin blood flowmotion in subjects with familial predisposition or newly diagnosed hypertension," Blood Press, vol. 19, no. 6, pp. 366-72, Dec, 2010.

[36] G. J. Hodges, and A. T. Del Pozzi, "Noninvasive examination of endothelial, sympathetic, and myogenic contributions to regional differences in the human cutaneous microcirculation," Microvasc Res, vol. 93, pp. 87-91, May, 2014.

[37] A. T. Del Pozzi, J. T. Miller, and G. J. Hodges, "The effect of heating rate on the cutaneous vasomotion responses of forearm and leg skin in humans," Microvasc Res, vol. 105, pp. 77-84, May, 2016.

[38] D. Balaz, A. Komornikova, P. Kruzliak, P. Sabaka, L. Gaspar, A. Zulli, M. Kucera, V. Zvonicek, J. Sabo, E. Ambrozy, and A. Dukat, "Regional differences of vasodilatation and vasomotion response to local heating in human cutaneous microcirculation," Vasa, vol. 44, no. 6, pp. 458-65, Nov, 2015.

[39] D. L. Kellogg, Jr., "In vivo mechanisms of cutaneous vasodilation and vasoconstriction in humans during thermoregulatory challenges," J. Appl Physiol, vol. 100, no. 5, pp. 1709-1718, 5/2006, 2006.

[40] M. Aboy, R. Hornero, D. Abasolo, and D. Alvarez, "Interpretation of the Lempel-Ziv complexity measure in the context of biomedical signal analysis," IEEE Trans Biomed Eng, vol. 53, no. 11, pp. 2282-8, Nov, 2006.

[41] Y. Zhang, S. Wei, H. Liu, L. Zhao, and C. Liu, "A novel encoding Lempel-Ziv complexity algorithm for quantifying the irregularity of physiological time series," Comput Methods Programs Biomed, vol. 133, pp. 7-15, Sep, 2016.

[42] A. Lempel, and J. Ziv, "On the Complexity of Finite Sequences," IEEE Transactions on Information Theory, vol. IT-22, no. 1, pp. 75-81, 1976.

[43] J. T. Butcher, A. G. Goodwill, S. C. Stanley, and J. C. Frisbee, "Blunted temporal activity of microvascular perfusion heterogeneity in metabolic syndrome: a new attractor for peripheral vascular disease?," Am. $J$. Physiol Heart Circ. Physiol, vol. 304, no. 4, pp. H547-H558, 2/15/2013, 2013.

[44] R. K. Pradhan, and V. S. Chakravarthy, "A computational model that links non-periodic vasomotion to enhanced oxygenation in skeletal muscle," Math Biosci, vol. 209, no. 2, pp. 486-99, Oct, 2007.

[45] D. L. Kellogg, Jr., Y. Liu, I. F. Kosiba, and D. O'Donnell, "Role of nitric oxide in the vascular effects of local warming of the skin in humans," $J$. Appl Physiol, vol. 86, no. 4, pp. 1185-1190, 4/1999, 1999.

[46] C. J. Smith, L. Santhanam, R. S. Bruning, A. Stanhewicz, D. E. Berkowitz, and L. A. Holowatz, "Upregulation of inducible nitric oxide synthase contributes to attenuated cutaneous vasodilation in essential hypertensive humans," Hypertension, vol. 58, no. 5, pp. 935-42, Nov, 2011.

[47] R. S. Bruning, L. Santhanam, A. E. Stanhewicz, C. J. Smith, D. E. Berkowitz, W. L. Kenney, and L. A. Holowatz, "Endothelial nitric oxide synthase mediates cutaneous vasodilation during local heating and is attenuated in middle-aged human skin," J Appl Physiol (1985), vol. 112, no. 12, pp. 2019-26, Jun, 2012. 